\title{
Adherence to Recommendations and Quality of Endoscopic Colorectal Cancer Surveillance in Long-Standing Ulcerative Colitis
}

\author{
Giulia Santi $^{a}$ Pierre Michetti $^{\text {b, e }}$ Florian Froehlich ${ }^{c}$ Jean-Benoît Rossel ${ }^{d}$ \\ Valérie Pittet $^{d} \quad$ Michel H. Maillard ${ }^{b}$ e Swiss IBD Cohort Study Group \\ aFaculty of Biology \& Medicine, University of Lausanne, Lausanne, Switzerland; ${ }^{b}$ Crohn's \& Colitis Center, \\ Gastroentérologie Beaulieu SA, Lausanne, Switzerland; 'Division of Gastroenterology \& Hepatology, Basel \\ University Hospital, Basel, Switzerland; ' ${ }^{\mathrm{C}}$ Center for Primary Care \& Public Health, University of Lausanne, Lausanne, \\ Switzerland; eService of Gastroenterology \& Hepatology, Lausanne University Hospital, Lausanne, Switzerland
}

\section{Keywords \\ Colonoscopy · Colorectal cancer · Early detection · \\ Ulcerative colitis · International guidelines}

\begin{abstract}
Background: Long-standing ulcerative colitis has been associated with an increased risk of colorectal cancer (CRC). Current guidelines recommend endoscopic CRC screening after 8 years of disease duration. The objectives of our study were to assess the adherence to recommendations and the quality of endoscopic procedure in long-standing ulcerative colitis. Methods: This is a retrospective cohort study. We selected patients included in the Swiss IBD cohort with a disease duration of $\geq 8$ years and an extension above the rectosigmoid junction. The complementary medical chart review focused on endoscopy and associated histological reports in 8 Swiss centers. Descriptive analyses focused on patients and their colonoscopies. Results: 309 colonoscopies were conducted among 116 patients with the following characteristics: women 47\%, mean age at diagnosis 31 years, and pancolitis disease extent in $65.5 \%$ of cases; $38.8 \%$ of patients had a first screening colonoscopy $<8$ years, $13.8 \%$ between 8 and 10 years, and $47.4 \%>10$ years. Cecal intubation was
\end{abstract}

karger@karger.com www.karger.com/iid

Karger $\frac{1}{\%}$

GOPEN ACCESS
(C) 2020 The Author(s)

Published by S. Karger AG, Basel

This is an Open Access article licensed under the Creative Commons Attribution-NonCommercial-4.0 International License (CC BY-NC) (http://www.karger.com/Services/OpenAccessLicense), applicable to the online version of the article only. Usage and distribution for commercial purposes requires written permission. performed in $94.5 \%$ of cases, and bowel preparation was good to excellent in $61.5 \%$ of endoscopies. Chromoendoscopy was used in $7.4 \%$ of cases, and the mean withdrawal time was 16.4 min. Dysplasia was found in $6.2 \%$ of cases. Conclusion: Despite current international recommendations, a significant number of patients did not receive a proper endoscopic surveillance. An increased use of chromoendoscopy, monitoring of withdrawal time, and appropriate bowel preparation would increase the quality of CRC screening. The adherence to screening guidelines and endoscopic quality should be promoted and standardized.

(c) 2020 The Author(s)
Published by S. Karger AG, Basel

\section{Introduction}

Colorectal cancer (CRC) is estimated to affect $5-6 \%$ of the global population in Europe and the USA. The incidence in Switzerland is around 4,000 new cases/year, and

Swiss IBD Cohort Study Group: see "Appendix" for detailed list of authors. 
it is considered to be a major cause of death in 1,600 individuals/year [1].

Inflammatory bowel disease (IBD) is the third most significant condition that increases the overall risk of developing $\mathrm{CRC}$, after familial adenomatous polyposis (FAP) and Lynch syndrome. The ulcerative colitis (UC)associated CRC risk depends on disease duration and differs from that of the general population after 8-10 years [2]: the literature describes a risk of developing CRC of $>2-5$ times in IBD patients compared to the general population of the same age group [3-6]. The probability of developing CRC in UC patients is estimated to be $2 \%$ at 10 years, $8 \%$ at 20 years, and $18 \%$ at 30 years, regardless of disease extent [7]; however, more recent studies observed a lower incidence of $2.5 \%$ at 20 years and $7.6 \%$ at 30 years [8]. Disease severity also has an effect on cancer risk: uncontrolled inflammation was demonstrated to increase the cancer risk in UC patients [2,9]. Other factors associated with a greater risk of cancer include concomitant primary sclerosing cholangitis (PSC), disease extent, family history, age, and presence of postinflammatory polyps $[4,9,10]$. In particular, PSC is strongly associated with IBD, especially with UC, with an increased risk of early CRC with a poor prognosis $[11,12]$.

This risk stratification for UC implicates modification of the timing of endoscopic surveillance. At present, colonoscopic surveillance is considered to be the best preventive strategy against CRC development in UC patients [2, $3,13]$. In order to reduce CRC morbidity and mortality, regular endoscopic follow-up is recommended [9]. European Crohn's and Colitis Organisation (ECCO) 2013 and British Society of Gastroenterology (BSG) 2010 guidelines recommend a first colonoscopy $8-10$ years after disease onset, except in presence of proctitis or Crohn's colitis involving just one segment of the colorectum. If no dysplasia is found during the first colonoscopy, screening intervals will be determined and planned every $1-5$ years, according to the risk stratification $[10,14]$. The American Gastroenterology Association (2010) suggests beginning the surveillance 8 years after the onset of the disease with a follow-up scheduled every 1-3 years [15].

In UC-PSC patients, the greater risk justifies the necessity for endoscopic surveillance, with a yearly colonoscopy starting at PSC diagnosis $[10,16]$. Ananthakrishnan et al. [17], in a retrospective study involving 6,823 IBD patients, found that having had a colonoscopy in the past 3 years reduced the CRC incidence rate from 2.7 to $1.6 \%$; furthermore, a reduced rate of mortality was found among those patients diagnosed with CRC who had had a colonoscopy in the last 6-36 months before diagnosis [17]. In our study, we hypothesize that CRC screening in patients with long-standing UC might be inefficient because of limited adherence to international recommendations. The main aim of this study was therefore to assess whether CRC screening of patients with long-standing UC was widely performed in clinical practice and to describe when and how it was performed. We also analyzed the technical characteristics used for the endoscopy together with the endoscopic and pathologic findings.

\section{Methods}

Study Design

We conducted a retrospective study among adult patients enrolled in the Swiss IBD Cohort Study (SIBDCS) [18]. The SIBDCS is a Swiss National Science Foundation (SNSF)-supported research initiative that comprises $>3,500$ patients followed up since 2006. This study is conducted under the approval of the ethics committee of University of Zurich that approved the SIBDCS.

\section{Study Population}

We included all UC patients with a disease duration of at least 8 years and a documented disease extension above the rectosigmoid junction and thus considered to be at moderate-to-high risk of developing CRC. We excluded patients having concomitant sclerosing cholangitis. Other exclusion criteria were presence of stenosis and/or fistula(e) and history of CRC.

\section{Data Extraction}

The following data were extracted from the SIBDCS databases: patient characteristics (age, gender, and disease duration), prior and current medications (immunomodulators, biologics, and 5-aminosalicylates), disease extent, colonoscopic findings, and screening intervals. Endoscopic and histopathologic details were retrieved from medical records in 8 Swiss centers ( 5 university hospitals and 3 private practices). We only considered endoscopies conducted after the moment of diagnosis. We collected informations on the indication for the procedure (CRC screening or disease control). We referred to the European guidelines (ECCO 2013) as they are the main reference in Switzerland [10]. Endoscopic quality was assessed through the collection of the following variables: cecal intubation (yes/no), ileal intubation (yes/no), withdrawal time, use of dye-based chromoendoscopy or virtual chromoendoscopy, and number of biopsies taken. Finally, we also collected findings from screening exams.

\section{Statistical Analyses}

The aforementioned characteristics are categorical data. We present them by using raw frequencies and percentages. Continuous data were summarized by their mean and their standard deviation. We analyzed the timing from diagnosis to the first screening colonoscopy through a histogram and a cumulative distribution function. Statistical analyses were performed with STATA 14 software (StataCorp, 2015, College Station, TX, USA).
Santi et al. 


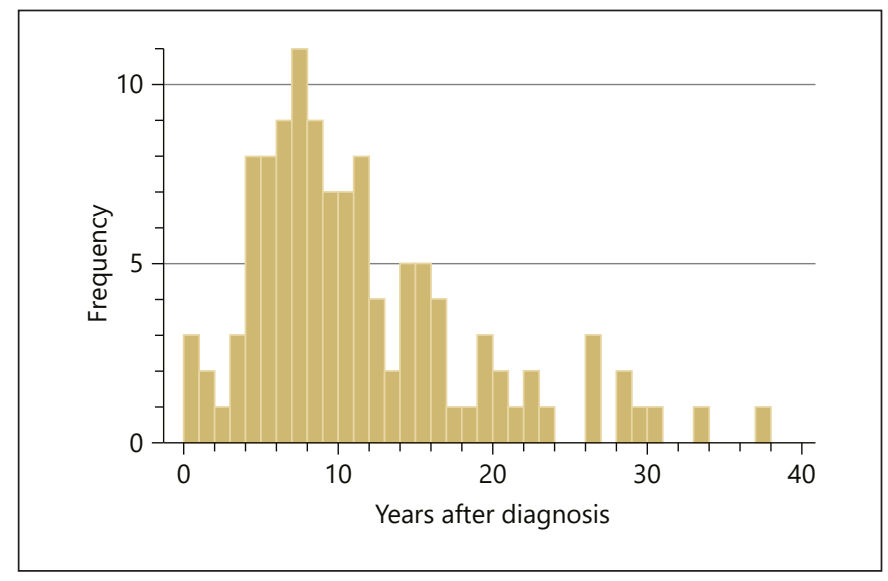

Fig. 1. Time between UC diagnosis and the first screening colonoscopy. Each bar shows the number of patients (frequency) having undergone a screening colonoscopy at the specific time point. UC, ulcerative colitis.

\section{Results}

\section{Patient Characteristics}

A total of 116 patients and 309 colonoscopies were included in the study. The mean age at diagnosis was 31 years (SD 10.4), and $47 \%(n=54)$ of patients were women. Disease extent was pancolitis in $65.5 \%$ of patients $(n=$ 76). After 8 years of disease duration, almost all patients had received 5-ASA $(n=108 ; 93 \%), 69 \%(n=80)$ received immunomodulators, and $37 \%(n=43)$ received biologic agents. The indication for endoscopy (Table 1 ) was CRC surveillance in $77 \%$ of the colonoscopies $(n=238)$, of which half were conducted when the disease was in remission and 38\% when it was active. Endoscopies were performed for disease control in a fifth of the cases.

\section{Time to First Surveillance Colonoscopy}

The mean time between UC diagnosis and the first screening colonoscopy performed for CRC screening or disease control was 11.5 years (SD 7.4; median 9.4 years). The time between UC diagnosis and the first screening colonoscopy was $<8$ years after diagnosis for 45 patients (38.8\%), between 8 and 10 years for 16 patients (13.8\%), and after $>10$ years for 55 patients (47.4\%) (Fig. 1). By looking at the cumulative distribution of this time, we observed that the first screening colonoscopy was conducted in $<10$ years for $53 \%$ of patients $(n=61)$ (Fig. 2$)$. We then hypothesized that left-sided colitis patients might be screened later due to less extensive disease. To address this point, we compared the screening interval between



Fig. 2. Cumulative distribution function of the time between UC diagnosis and the first screening colonoscopy. UC, ulcerative colitis.

Table 1. Characteristics of the 309 endoscopies performed in the 116 patients included in the study

$n(\%)$

Indication for colonoscopy

CRC screening

Disease control

$238(77.0)$

Unknown

$7(2.3)$

Disease activity in CRC screening colonoscopies

Remission

$132(55.5)$

Active disease

$92(20.7)$

Unknown

$14(5.9)$

patients with pancolitis and those with left-sided colitis. The median interval for pancolitis was 9.3 years $(n=76$; $\mathrm{IQR}=6.1-14.0$, range $=0.5-37.3)$, while it was 9.7 years for the left-sided colitis $(n=40$; IQR $=7.1-16.1$, range $=$ 3.4-33.7). This indicates that patients with left-sided colitis are screened at similar intervals than patients with pancolitis.

\section{Endoscopic Quality Indicators}

Reviewing the 309 endoscopic reports revealed a proportion of cecal intubation of $94.5 \%(n=292)$ and of ileal intubation of $84.1 \%(n=260)$. Quality of bowel preparation was documented in $70.2 \%$ of the exams. When documented, bowel preparation was reported as good to 


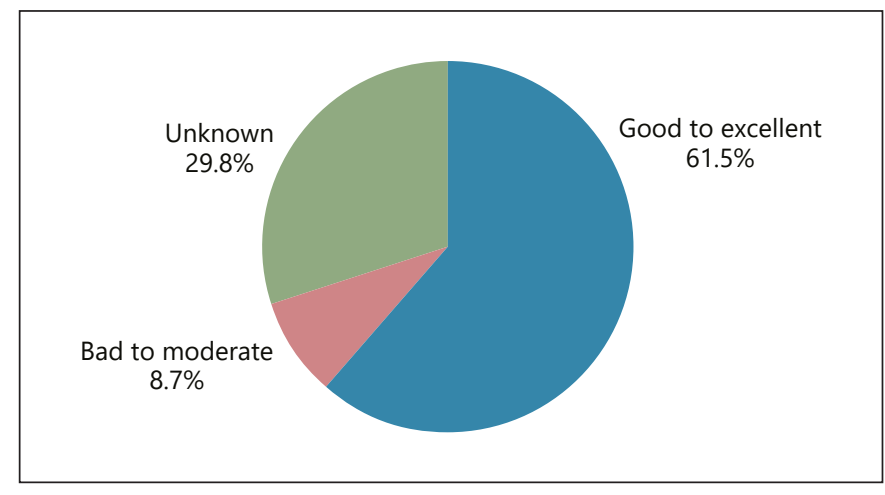

Fig. 3. Quality of bowel preparation for the 309 endoscopic procedures.

Table 2. Quality indicators of screening colonoscopies $(n=309)$

\begin{tabular}{lc}
\hline & $N(\%)$ \\
\hline Cecal intubation & $292(94.5)$ \\
Ileal intubation & $260(84.1)$ \\
Mean (SD) withdrawal time & $16.4(11.5)$ \\
Chromoendoscopy & \\
$\quad$ Yes & $23(7.4)$ \\
$\quad$ No & $270(87.4)$ \\
$\quad$ Unknown & $16(5.2)$ \\
Virtual chromoendoscopy & \\
$\quad$ Yes & $48(15.5)$ \\
$\quad$ No & $247(79.9)$ \\
Unknown & $14(4.5)$ \\
$\quad$ Mean number (SD) of biopsies taken during & $19.3(15.3)$ \\
\hline
\end{tabular}

excellent in 190 procedures and bad to moderate in 27 (Fig. 3). Withdrawal time was documented in 37 colonoscopies $(12.0 \%)$ with a mean of $16.4 \mathrm{~min}$ (SD 11.5). Chromoendoscopy was used in $7.4 \%$ of the procedures $(n=23)$ and virtual chromoendoscopy (NBI, FICE, or iScan) in $15.5 \%$. The mean number of biopsies taken during whitelight colonoscopy was 19.3 (SD 15). The total number of dysplasia observed in random and target biopsies is 19, corresponding to $6.2 \%$ of all colonoscopies carried out (Table 2). No cancers were found.

\section{Discussion}

In this study, we were interested in exploring whether CRC surveillance was conducted in long-standing UC patients at moderate-to-severe risk and whether gastroenter- ologists followed the guidelines in terms of time to screening endoscopy procedures. We found some differences betweendailypracticeand theinternationalrecommendations.

Overall, surveillance colonoscopies should be conducted during a disease remission phase, as ongoing inflammation raises the risk of higher false-positive rates for dysplasia detection. Nevertheless, in patients with chronic long-standing severe UC, screening may be performed considering the risk stratification, as those patients have a high risk of developing cancer $[10,14,19]$. International guidelines (ECCO and BSG) consider the starting point of colonoscopic surveillance should be set at 8-10 years after diagnosis, given that the CRC-UC risk depends on disease duration and differs from that of the general population after this time. In our study, the explicit indication for the first screening colonoscopy was mentioned in $77.9 \%$ of the procedures. Only $52.6 \%$ of all colonoscopies were performed $<10$ years after diagnosis. Altogether, those data demonstrate that surveillance colonoscopy was only performed in a subset of patients. It also showed that a large number of patients had a screening colonoscopy during the active phase of the disease, which hampered the expected state-of-the-art surveillance procedures. In our study, the screening interval between patients with pancolitis (9.3 years) and those with left-sided colitis (9.7 years) was similar.

The effectiveness of colonoscopy in order to reduce CRC incidence and mortality largely depends on the quality of the endoscopic exam [20]. Some quality indicators include bowel preparation quality, withdrawal time, cecal intubation rate, endoscopist's level of expertise, and adenoma detection rate (ADR). ADR is defined as the number of colonoscopies, in which at least one polyp is found, divided by the total number of surveillance colonoscopies performed [21, 22]. The American Society for Gastrointestinal Endoscopy recommends that an overall ADR of $>25 \%$ (30\% for men and $20 \%$ for women) should be achieved [23].

Guidelines from the European Society of Gastrointestinal Endoscopy (ESGE) suggest that cecal and ileal intubation should occur in at least $90 \%$ of all screening colonoscopies for CRC [24]. In our population, cecal and ileal intubation was conducted in $94.5 \%$ and $84.1 \%$ of cases, respectively. Quality of bowel preparation is another important indicator of efficient endoscopic surveillance. Current guidelines recommend that adequate preparation should be obtained in at least $90 \%$ of all surveillance colonoscopies [24]. A retrospective study conducted on 12,787 patients showed that suboptimal bowel preparation had a significant impact on the rate of miss-
28

Inflamm Intest Dis 2021;6:25-31 DOI: $10.1159 / 000511010$
Santi et al. 
ing adenoma. There is however no clear recommendation on the correct interval to the next endoscopy after a suboptimal bowel preparation [25]. In the present study, good-to-excellent preparation was reported in $61.5 \%$ of endoscopies, while documentation was missing for $29.8 \%$ of the procedures. Overall, these data suggest that bowel preparation should be optimized in this patient population and that endoscopic reports should be improved to better document this quality indicator.

Endoscope withdrawal time has been described as a meaningful quality indicator for surveillance colonoscopy. Guidelines (ESGE) suggest a time of at least 6 min in 90\% of endoscopies [24]. In our study, the mean withdrawal time was found to be close to $16 \mathrm{~min}$. However, we noticed that this parameter was very rarely documented in the reports, suggesting that few gastroenterologists are aware of the importance of this indicator. Although there was a low rate of reporting of these data, our results suggest that withdrawal time was prolonged when performing surveillance colonoscopy in a UC patient population. Putative reasons for this could be the use of chromoendoscopy or image filtering techniques such as FICE, NBI, or iScan.

Compared to a few years ago, random biopsy sampling (4 random biopsies every $10 \mathrm{~cm}$ ) is currently debated as a first-choice strategy while targeted biopsies under chromoendoscopy arises as the gold standard. Although some studies suggest that dysplasia detection might be comparable [26], others demonstrate that random sampling is more lengthy, associated with lower diagnostic yield, and less cost-effective [27, 28].

For those reasons, European guidelines recommend the use of surface enhancement imaging strategies with targeted biopsies [29]. The surface enhancement strategy can be performed using dye-spraying methods such as indigo carmine or methylene blue or virtually via image filtering techniques. Indeed, recent data suggest that image filtering techniques are not inferior to chromoendoscopy, while the latter was associated with a longer withdrawal time $[26,28,30]$. In our study, excluding colonoscopies in which chromoendoscopy was performed, the mean number of colonic biopsies was 19.3 (SD 15.3), while this should have been of at least 40 . We suppose that every center used high-definition scopes. Chromoendoscopy was performed in 23 colonoscopies (total 7.44\%), and virtual chromoendoscopy was used in slightly $>1 / 6$ endoscopies. Although our study does not allow us to distinguish between chromoendoscopy underuse and inappropriate reporting, it suggests that endoscopists should pay more attention to report and use surface enhancement imaging strategies for screening colonoscopies.

Colorectal Cancer Surveillance in Ulcerative Colitis
On the practical side, we suggest that a standardization of colonoscopy reports would simplify the work of the operators; at the same time, improved education of patients and doctors concerning current international guidelines could increase compliance and practical outcomes. This screening strategy is suggested by international guidelines (ECCO, AGA, and BSG) and supported by the current literature; however, in our opinion, it deserves further study and investigation in order to strengthen the evidence and increase its effectiveness in clinical practice.

Despite the large body of evidence arguing for endoscopic surveillance in long-standing UC, several factors might influence patients' and doctors' adherence to these recommendations. For example, complete mucosal healing is mandatory in order to avoid bias in histological analysis and detection of dysplasia. In this regard, we mention the utility of a calprotectin test prior to screening colonoscopy to increase the likelihood to conduct the screening exam in a mucosal healing state. However, healing was not frequently obtained in the present study despite adequate medical management. In addition, as most UC patients undergo several endoscopic procedures for disease follow-up and detection of flares, they might be reluctant to undergo a colonoscopy when in remission. Few studies deal with the psychosocial impact of CRC surveillance in IBD patients, but one in particular shows that there is no impact on quality of life of patients included in a CRC surveillance program [31].

Our study has several limitations. First, this is a retrospective study with a small sample size of patients and colonoscopies with all the analyses being based on nonstandardized medical charts, which may be prone to reporting bias. In addition, differences in reporting and clinical practices could technically exist between hospital and private centers. Given the limited sample size, those differences could not be addressed. Concerning the activity of the disease, we observed that a large number of endoscopies revealed active histological and endoscopic activity despite clinical remission. In addition, the timing of an endoscopic exam might influence the technical practice (chromoendoscopy having been quite recently advocated as a preferred screening method).

\section{Conclusion}

Despite current international recommendations, a significant number of UC patients did not receive an adequate endoscopic surveillance in terms of timing and 
Table 3. List of parameters that should be included in a standardized endoscopy report for UC-associated colorectal cancer surveillance

Initial disease extent

Current medication

Disease activity (Mayo or UCEIS score)

Cecal intubation, yes/no

Ileal intubation, yes/no

Withdrawal time

Chromoendoscopy, yes/no

Virtual chromoendoscopy, yes/no

Type of bowel preparation

Preparation quality (Boston score)

quality. In addition, our study indicates that the quality of endoscopic exams was insufficiently documented, chromoendoscopy was seriously underused, and random biopsies were insufficient when associated with whitelight endoscopy. Further studies to better understand the lack of adherence to screening recommendations and further instruction for practicing gastroenterologists should be organized to improve the endoscopic surveillance of patients with long-standing UC (Table 3 ).

\section{Appendix}

Members of the SIBDCS group:

Karim Abdelrahman, Gentiana Ademi, Patrick Aepli, Amman Thomas, Claudia Anderegg, Anca-Teodora Antonino, Eva Archanioti, Eviano Arrigoni, Nurullah Aslan, Diana Bakker de Jong, Bruno Balsiger, Mamadou-Pathé Barry, Polat Bastürk, Peter Bauerfeind, Andrea Becocci, José M. Bengoa, Luc Biedermann, Janek Binek, Mirjam Blattmann, Stephan Boehm, Tujana Boldanova, Jan Borovicka, Christian P. Braegger, Stephan Brand, Francisco Bravo, Lukas Brügger, Simon Brunner, Patrick Bühr, Sabine Burk, Emanuel Burri, Matthias Butter, Sophie Buyse, Dahlia-Thao Cao, Ove Carstens, Dominique H. Criblez, Fabrizia D’Angelo, Philippe de Saussure, Lukas Degen, Joakim Delarive, Christopher Doerig, Barbara Dora, Susan Drerup, Carole Ducrey, Ali El-Wafa, Matthias Engelmann, Aude Erdmann-Voisin, Christian Felley, Markus Fliegner, Montserrat Fraga, Yannick Franc, Pascal Frei, Remus Frei, Michael Fried, Florian Froehlich, Raoul Ivano Furlano, Luca Garzoni, Martin Geyer, Marc Girardin, Delphine Golay, Ignaz Good, Ulrike Graf Bigler, Sébastien Godat, Thomas Greuter, Beat Gysi, Johannes Haarer, Marcel Halama, Janine Haldemann, Pius Heer, Benjamin Heimgartner, Beat Helbling, Peter Hengstler, Denise Herzog, Cyrill Hess, Roxane Hessler, Klaas Heyland, Thomas Hinterleitner, Claudia Hirschi, Petr Hruz, Pascal Juillerat, Ioannis Kapoglou, Stephan Kayser, Céline Keller, Carolina Khalid-de Bakker, Christina Knellwolf, Christoph Knoblauch, Henrik Köhler, Rebekka Koller, Claudia Krieger, Patrizia Künzler, Rachel Kusche, Frank Serge Lehmann, Andrew Macpherson, Michel H. Maillard, Michael Manz, Maude Martinho, Rémy Meier, Christa Meyenberger, Pamela Meyer, Pierre Michetti, Benjamin Misselwitz,
Bernhard Morell, Patrick Mosler, Eleni Moschouri, Christian Mottet, Christoph Müller, Beat Müllhaupt, Leilla Musso, Michaela Neagu, Cristina Nichita, Jan Niess, Andreas Nydegger, Nicole Obialo, Cassandra Oropesa, Ulrich Peter, Daniel Peternac, Laetitia Marie Petit, Valérie Pittet, Daniel Pohl, Marc Porzner, Claudia Preissler, Nadia Raschle, Ronald Rentsch, Sophie Restellini, JeanPierre Richterich, Sandra Riedmüller, Branislav Risti, Marc Alain Ritz, Gerhard Rogler, Nina Röhrich, Jean-Benoît Rossel, René Roth, Vanessa Rueger, Markus Sagmeister, Gaby Saner, Riad Sarraj, Bernhard Sauter, Mikael Sawatzki, Michael Scharl, Sylvie Scharl, Martin Schelling, Susanne Schibli, Hugo Schlauri, Dominique Schluckebier, Daniela Schmid, Sybille Schmid, Jean-François Schnegg, Alain Schoepfer, Philipp Schreiner, Frank Seibold, Mariam Seirafi, Gian-Marco Semadeni, Arne Senning, Christiane Sokollik, Joachim Sommer, Johannes Spalinger, Holger Spangenberger, Philippe Stadler, Peter Staub, Dominic Staudenmann, Volker Stenz, Michael Steuerwald, Alex Straumann, Andreas Stulz, Michael Sulz, Michela Tempia-Caliera, Joël Thorens, Kaspar Truninger, Radu Tutuian, Patrick Urfer, Stephan Vavricka, Francesco Viani, Fabrizion Vinzens, Jürg Vögtlin, Roland Von Känel, Dominique Vouillamoz, Rachel Vulliamy, Marianne Vullièmoz, Paul Wiesel, Reiner Wiest, Stefanie Wöhrle, Bahtiyar Yilmaz, Samuel Zamora, Silvan Zander, Jonas Zeitz, and Dorothee Zimmermann.

\section{Acknowledgements}

The authors are grateful for the support and the contribution of all the study nurses of the SIBDCS and thank the teams of the gastroenterology departments of Hôpitaux Universitaires de Genève (HUG), Center hospitalier universitaire vaudois (CHUV), Inselspital-Universitätsspital Bern, Universitätsspital Zürich (USZ), Universitätsspital Basel, and of the private practices that participated in the study.

\section{Statement of Ethics}

Ethics approval was obtained from the regional Swiss Ethics Committees in which SIBDCS cohort participants were enrolled (Commission d'éthique du Canton de Vaud/Protocol No. 33/06). Written informed consent was obtained from each patient included in the study. The study was conducted in accordance with the World Medical Association Declaration of Helsinki.

\section{Conflict of Interest Statement}

The authors have no conflicts of interest to declare.

\section{Funding Sources}

This study was supported by the Swiss National Science Foundation (SNSF) (Grant No. 33CS30-148422) (Swiss IBD cohort study).
Santi et al. 


\section{Author Contributions}

Giulia Santi, Michel H Maillard, and Valérie Pittet realized the study design and the data collection from medical records in the 8 Swiss centers. Jean-Benoît Rossel and Valérie Pittet performed the data extraction from the SIBDCS databases and performed the statistical analysis. Pierre Michetti and Florian Froehlich were involved in planning and supervising the project. All authors contributed to the writing of the manuscript.

\section{References}

1 Dehlavi A, Nichita C, Brondolo VK, Bertolini D, Dorta G. Prevention of colorectal cancer. Rev Med Suisse. 2011;7(307):1704-9.

2 Andersen NN, Jess T. Has the risk of colorectal cancer in inflammatory bowel disease decreased? World J Gastroenterol. 2013;19(43): 7561-8.

3 Itzkowitz SH, Harpaz N. Diagnosis and management of dysplasia in patients with inflammatory bowel diseases. Gastroenterology. 2004;126(6):1634-48.

4 Beaugerie L, Itzkowitz SH. Cancers complicating inflammatory bowel disease. $\mathrm{N}$ Engl J Med. 2015;372(15):1441-52.

5 Scarpa M, Castagliuolo I, Castoro C, Pozza A, Scarpa M, Kotsafti A, et al. Inflammatory colonic carcinogenesis: a review on pathogenesis and immunosurveillance mechanisms in ulcerative colitis. World J Gastroenterol. 2014;20(22):6774-85.

6 Viennot S, Deleporte A, Moussata D, Nancey S, Flourié B, Reimund JM. Colon cancer in inflammatory bowel disease: recent trends, questions and answers. Gastroenterol Clin Biol. 2009;33(Suppl 3):S190-201.

7 Eaden JA, Abrams KR, Mayberry JF. The risk of colorectal cancer in ulcerative colitis: a meta-analysis. Gut. 2001;48(4):526-35.

8 Rutter MD, Saunders BP, Wilkinson KH, Rumbles S, Schofield G, Kamm MA, et al. Thirty-year analysis of a colonoscopic surveillance program for neoplasia in ulcerative colitis. Gastroenterology. 2006;130(4):1030-8.

9 Herszényi L, Barabás L, Miheller P, Tulassay Z. Colorectal cancer in patients with inflammatory bowel disease: the true impact of the risk. Dig Dis. 2015;33(1):52-7.

10 Annese V, Daperno M, Rutter MD, Amiot A, Bossuyt P, East J, et al. European evidence based consensus for endoscopy in inflammatory bowel disease. J Crohns Colitis. 2013; 7(12):982-1018.

11 Loftus EV Jr, Harewood GC, Loftus CG, Tremaine WJ, Harmsen WS, Zinsmeister AR, et al. PSC-IBD: a unique form of inflammatory bowel disease associated with primary sclerosing cholangitis. Gut. 2005;54(1):91-6.

12 Rossi RE, Conte D, Massironi S. Primary sclerosing cholangitis associated with inflammatory bowel disease: an update. Eur J Gastroenterol Hepatol. 2016;28(2):123-31.
13 Lutgens MW, Oldenburg B, Siersema PD, van Bodegraven AA, Dijkstra G, Hommes DW, et al. Colonoscopic surveillance improves survival after colorectal cancer diagnosis in inflammatory bowel disease. Br J Cancer. 2009; 101(10):1671-5.

14 Cairns SR, Scholefield JH, Steele RJ, Dunlop MG, Thomas HJ, Evans GD, et al. Guidelines for colorectal cancer screening and surveillance in moderate and high risk groups (update from 2002). Gut. 2010;59(5):666-89.

15 Farraye FA, Odze RD, Eaden J, Itzkowitz SH, McCabe RP, Dassopoulos T, et al. AGA medical position statement on the diagnosis and management of colorectal neoplasia in inflammatory bowel disease. Gastroenterology. 2010;138(2):738-45.

16 Tsaitas C, Semertzidou A, Sinakos E. Update on inflammatory bowel disease in patients with primary sclerosing cholangitis. World J Hepatol. 2014;6(4):178-87.

17 Ananthakrishnan AN, Cagan A, Cai T, Gainer VS, Shaw SY, Churchill S, et al. Colonoscopy is associated with a reduced risk for colon cancer and mortality in patients with inflammatory bowel diseases. Clin Gastroenterol Hepatol. 2015;13(2):322-e1.

18 Pittet V, Michetti P, Mueller C, Braegger CP, von Känel R, Schoepfer A, et al. Cohort profile update: the Swiss Inflammatory Bowel Disease Cohort Study (SIBDCS). Int J Epidemiol. 2019;48(2):385-6f

19 Rutter M, Saunders B, Wilkinson K, Rumbles S, Schofield G, Kamm M, et al. Severity of inflammation is a risk factor for colorectal neoplasia in ulcerative colitis. Gastroenterology. 2004;126(2):451-9.

20 Rex DK, Bond JH, Winawer S, Levin TR, Burt RW, Johnson DA, et al. Quality in the technical performance of colonoscopy and the continuous quality improvement process for colonoscopy: recommendations of the U.S. Multi-Society Task Force on Colorectal Cancer. Am J Gastroenterol. 2002;97(6):1296308.

21 Anderson JC, Butterly LF. Colonoscopy: quality indicators. Clin Transl Gastroenterol. 2015;6(2):e77.

22 Chaptini L, Laine L. Can I improve my adenoma detection rate? J Clin Gastroenterol. 2015;49(4):270-81.
23 Rex DK, Schoenfeld PS, Cohen J, Pike IM, Adler DG, Fennerty MB, et al. Quality indicators for colonoscopy. Am J Gastroenterol. 2015;110(1):72-90.

24 Rembacken B, Hassan C, Riemann JF, Chilton A, Rutter M, Dumonceau JM, et al. Quality in screening colonoscopy: position statement of the European Society of Gastrointestinal Endoscopy (ESGE). Endoscopy. 2012; 44(10):957-68.

25 Lebwohl B, Kastrinos F, Glick M, Rosenbaum AJ, Wang T, Neugut AI. The impact of suboptimal bowel preparation on adenoma miss rates and the factors associated with early repeat colonoscopy. Gastrointest Endosc. 2011; 73(6):1207-14.

26 Mooiweer E, van der Meulen-de Jong AE, Ponsioen CY, Fidder HH, Siersema PD, Dekker E, et al. Chromoendoscopy for surveillance in inflammatory bowel disease does not increase neoplasia detection compared with conventional colonoscopy with random biopsies: results from a large retrospective study. Am J Gastroenterol. 2015;110(7): 1014-21.

27 Rutter MD, Saunders BP, Schofield G, Forbes A, Price AB, Talbot IC. Pancolonic indigo carmine dye spraying for the detection of dysplasia in ulcerative colitis. Gut. 2004;53(2):25660.

28 Tontini GE, Vecchi M, Neurath MF, Neumann H. Review article: newer optical and digital chromoendoscopy techniques vs. dyebased chromoendoscopy for diagnosis and surveillance in inflammatory bowel disease. Aliment Pharmacol Ther. 2013;38(10):1198208.

29 Guagnozzi D, Lucendo AJ. Colorectal cancer surveillance in patients with inflammatory bowel disease: what is new? World J Gastrointest Endosc. 2012;4(4):108-16.

30 Bisschops R, Bessissow T, Joseph JA, Baert F, Ferrante M, Ballet V, et al. Chromoendoscopy versus narrow band imaging in UC: a prospective randomised controlled trial. Gut. 2018;67(6):1087-94.

31 Mountifield R, Bampton P, Prosser R, Mikocka-Walus A, Andrews JM. Colon cancer surveillance in inflammatory bowel disease: unclear gain but no psychological pain? Intern Med J. 2014;44(2):131-8. 